\title{
CLIL European-led Projects and Their Implications for Iranian EFL Context
}

\author{
Masoud Khalili Sabet ${ }^{1} \&$ Nima Sadeh ${ }^{1}$ \\ ${ }^{1}$ English Department, University of Guilan, Faculty of Humanities, University of Guilan, Rasht, Iran \\ Correspondece: Nima Sadeh, English Department, University of Guilan, Faculty of Humanities, University of \\ Guilan, Rasht, Iran. Tel: 98-911-134-0267. E-mail: nm_simple@yahoo.com
}

Received: September 2, 2011 Accepted: June 2, 2012 Online Published: July 27, 2012

doi:10.5539/elt.v5n9p88 URL: http://dx.doi.org/10.5539/elt.v5n9p88

\begin{abstract}
The main purpose of this paper is to examine the European-led projects in the field of Content and Language Integrated Learning (CLIL) and their potential applicability in the Iranian EFL context. This paper tries to introduce various dimensions of CLIL and examine the compatibility of its component with the Iranian context.
\end{abstract}

Keywords: Content and Language Integrated Learning, CLIL, Iranian EFL context

\section{Introduction}

CLIL (Content and Language Integrated Learning) is an educational approach within which non-language school or curriculum subjects are introduced through a foreign/second language instruction and communication context.

Scholars in the field of CLIL have offered various definitions for CLIL. The following are some of them:

'CLIL refers to situations where subjects, or parts of subjects, are taught through a foreign language with dual-focused aims, namely the learning of content, and the simultaneous learning of a foreign language'. (Marsh, 1994)

'CLIL is a generic term and refers to any educational situation in which an additional language, and therefore not the most widely used language of the environment, is used for the teaching and learning of subjects other than the language itself'. (Marsh \& Lange, 2000)

'CLIL is an educational approach in which non-language subjects are taught through a foreign, second or other additional language'. (Marsh et al, 2001)

'The use of languages learnt in the learning of other subjects'. (Lang, 2002)

'Integrating language with non-language content, in a dual-focused learning environment'. (Marsh, 2002)

According to Marsh\& Lange (2000) successful language learning takes place when people receive instruction (i.e. are taught) and at the same have the opportunity of using and experiencing language in authentic and real-life situations.

According to Marsh \& Lange (2000), though language classroom as an environment within which instruction is provided, is an essential part of the language learning process, in most cases based on the confinements of the classroom either in terms of time and resources, the teachers are not able to go beyond the classroom limits and manage to provide situations in which students really use language.

"What CLIL can offer to youngsters of any age, is a more natural situation for language development which builds on other forms of learning. This natural use of language can boost a youngster's motivation and hunger towards learning languages. It is this naturalness which appears to be one of the major platforms for CLIL'S importance and success in relation to both language and other subject learning”. (Marsh \& Lange, 2000)

Since the notion of CLIL is a recent one, it may take time for students to be familiarized with this type of education. However, once they get familiar with that it is highly likely that they improve at a higher rate than usual. Based on CILT, the National Center for Languages' website, students may benefit CLIL instructions from the following perspective:

- $\quad$ stronger links with the citizenship curriculum (particularly through the use of authentic materials, which offer an alternative perspective on a variety of issues) 
- $\quad$ increased student awareness of the value of transferable skills and

knowledge

- $\quad$ greater pupil confidence

Marsh and Lange (2000), taking the benefit of establishing the analogy between playing the piano and learning the language, mention that in case of non-language subjects there are almost always opportunities for learners to practically experience what they have already theoretically learned in a real context. For example a learner of the piano, after having received theoretical instructions, most probably is capable of finding an opportunity to practice what he/she has learned practically on a real piano. However, in the case of language, especially in EFL contexts, learners may rarely be capable of being benefitted from real-life and authentic opportunities to practically practice language. What Marsh and Lange try to suggest is that CLIL might possibly a highly reasonable solution to solve this problem. According to Marsh\& Lange (2000), the following are the potentials of CLIL approach:

- $\quad$ The ability to use a language is much more than knowing its words and grammar, and speaking in perfectly formed sentences. Central to language is ability to communicate which is a highly complex human behavior. Since the infants and very young children can demonstrate an incredible ability to communicate with a very limited knowledge of language, a learner of a second/foreign language may exhibit the ability of communicating even though the knowledge of grammar already acquired is faulty, the knowledge of word is weak or pronunciation ability is poor. This can be to a great extent fostered and enhanced through CLIL approach as it paves the way for real use of language.

- Learning different languages may greatly increase the learner's thinking abilities. Since CLIL is a dual-focused approach (content and language), it enhances the ability to think about different concepts in another language which itself double the thinking enhancement effects. CLIL provides the opportunity for the learner to learn to think in another language, not just the language itself as the main focus of the course.

- CLIL offers an opportunity to the youngsters to be actual bilingual or even pluralingual users of languages. CLIL can provide learners with this opportunity regardless of their social and economic positions. CLIL is a highly social phenomenon.

The primary goal of this study is to show the potential implications of European-led projects and researches in the field of CLIL in the Iranian context. As an EFL situation, Iranian learners of English virtually lose all their connection to authentic English outside the classroom environment i.e. the sole opportunity of practicing English is in the classroom. In this case, the major portion of educational burden is transferred to classes and school system.

According to Ghorbani (2009) local educational authorities in Iran have less power in decision-making as the educational policies are almost entirely decided by the central government and passed down to the local organization to be employed in schools. In this system the provincial schools are bound with the textbooks and methodologies determined by the superior Ministry of Education. As mentioned before, due the natural lack of authentic context of using English outside the classroom in such EFL context of Iran, it is expected that this lack is compensated by the educational policies within the school system. However, according to Jahangard (2007), Iranian students' oral and aural skills are not within the focus of Iranian EFL textbooks and the primary focus of the present educational system is to make students pass tests and exams. There also some other reasons that why Iranian students are not able to develop and subsequently demonstrate a reasonable level of proficiency in communication skills within the school framework. According to Namaghi (2006), there are some sociopolitical factors which affect the teaching process:

- Since the educational policies and consequently the educational materials are solely determined by a governmental organization- Ministry of Education- teachers cannot choose the materials which best suit their students' needs

- $\quad$ The output of teaching process is highly influenced by the national testing system. Thus the assessment scheme used by teachers may not go beyond the goals of the national testing system. As an evident consequence, in this way teachers are not able to develop tests which have positive washback effects on the learners.

- Since the Iranian context is highly sensitive to educational achievements which are almost always seen in the scores obtained by the students, there is a great pressure from parents, students and school authorities on teachers. In this case it seems impossible for a teacher to deviate what has already been determined by the central policymaker.

It is evident that within such a context neither the lack of authentic opportunities of foreign language is 
compensated by the Iranian educational system nor the educational system contributes to achieving even the minimal levels of proficiency in foreign language acquisition.

Based on the definitions and the potential advantages of incorporating CLIL approach into the national educational policies and the inefficiencies of Iranian educational system in providing an effective foreign language learning and acquisition context which have been hereinbefore mentioned, the author would like to propose that, based on the following reasons, establishing a movement toward incorporating CLIL approach in Iranian educational system is of evident benefits:

- Most youngsters spend at least 12 years in this educational system before entering academic environmentsthis can be increased if we take into account the preschool courses, which potentially provides the students with an invaluable opportunity to acquire a foreign language.

- CLIL approach perfectly works within the national educational system since it integrates the subject matters taught at schools and language.

- It may perfectly compensate the lack of authentic opportunities of foreign language use which is an inherent quality of any EFL context.

- It necessarily leads to nurturing students with higher international academic skills and better cross-cultural understanding.

\section{CLIL Dimensions and Focuses and Their Implication in Iranian Context}

According to the CLIL Compendium, a European project supported and funded by the Directorate-General for education and culture of the European commission, there are 5 dimensions based on issues relating to culture, environment, language, content and learning. Within each dimension there are a number of focal points based on three major factors: age-range of learners, sociolinguistic environment, and degree of exposure to CLIL. These factors will be fully discussed in the section relating to types of CLIL.

The 5 dimensions are as follow:

\subsection{The Culture Dimension - CULTIX}

\subsubsection{Build Intercultural Knowledge \& Understanding}

Iran is a developing country and is not needless of foreign technologies and resources. Therefore, the potentiality of establishing international relationship is significantly high as it has already been established to some extent. Another point is that, based on geographical diversity, Iran is a highly potential tourist destination. Though, as a result of relative isolation of Iran in international community in recent years and also religious limitations, this potential has not been turned into an actuality, it seems obvious that Iran will be benefitted from this global market soon or later. The establishment of such industry might be translated into an increasing number of tourists who in turn will need infrastructures like hotels and related industries. This emerging need will lead to a high demand of knowledgeable work force with high intercultural knowledge and understanding. Therefore, any investment in this field will be of evident benefits.

\subsubsection{Develop Intercultural Communication Skills}

As mentioned before, Iranian educational system has proved to fail to produce proficient users of English since its main focus is on national exams. Further, based on relative isolation of Iran in international community, the degree of exposure to different cultures is relatively low in Iran. Since incorporating CLIL methodologies into Iranian educational system will be in need of investigating and even emulating foreign resources, this trend will necessarily result in an introduction to different points of view employed in other countries in order to develop educational materials which in turn are true reflections of cultural atmospheres of those countries. Such a process will help the students and even teachers and other authorities develop better intercultural communication skills.

\subsubsection{Learn about Specific Neighboring Countries/Regions and/or Minority groups}

Since this project has been conducted in a European framework, this aspect of cultural dimension might not be relevant as such in Iranian context. However, this aspect may offer some insights. Incorporating CLIL approach will definitely result in better understanding of different countries even if not neighboring countries.

\subsubsection{Introduce the Wider Cultural Context}

Introducing a foreign language, specifically English into an educational system will bring with itself cultural issues related to that language and speakers of that language. Additionally, mixing language with specific contents will make students aware of other cultures' perspective toward life-related issues. 


\subsection{The Environment Dimension - ENTIX}

\subsubsection{Prepare for Internationalization, Specifically EU Integration}

Except the part for EU integration which is not relevant for Iran, studying in an international language, even if partly, will bring with itself a more thorough understanding of the international community. As mentioned earlier, Iran is on the verge of being international. Introducing CLIL approach is a strategic investment.

\subsubsection{Access International Certification}

Incorporating CLIL approach means being more in harmony with international standards. As a consequence of Iranian educational system a strong tendency toward certification has been created between Iranian students and even their parents. An increase in number of private institutes which offer courses to prepare students for entering different undergraduate courses, and even the permanent requests of Iranian English learners for a kind of certificate are symptoms Iranians' tendency toward certification. Integrating CLIL approach into this kind of educational system may correct these kind of views toward certification while providing a sound framework for international harmonization and certification.

\subsubsection{Enhance School Profile}

In recent years, English has been included in the programs of most private kindergartens and preschools in Iran. Growing interest in Iranian parents to send their children to these institutes despite of their relatively high expenses might be considered as a sign of understanding the position of English as an international language among Iranian parents. Subsequently, schools and institutes offering these kinds of courses are likely to find a higher prestige in the market. Further, regardless of social prestige, introducing CLIL courses in such institutes means entering more English proficient teachers which in turn means a possible higher understanding of international trends in education.

\subsection{The Language Dimension - LANTIX}

\subsubsection{Improve Overall Target Language Competence}

Since the emergence of the notion of language acquisition, huge amounts of study have been conducted in order to propose a workable framework within which language can be acquired more naturally and effectively. According to Marsh \& Lange (2000) successful language learning may occur when people alongside the instructions they receive can experience authentic and real-life situations in which they can use and acquire language. As mentioned before, in an EFL situation of Iran in which the connection to English and chance of having real-life opportunities to use English outside the classroom is virtually zero, creating a quasi authentic situation inside the classroom and within the school framework seems to be a rational remedy. If this is the case, introducing CLIL in the Iranian school system seems to be the best option.

\subsubsection{Develop Oral Communication Skills}

Based on what hereinbefore mentioned e.g. Ghorbani (2009), Iranian students' oral and aural skills are not within the focus of Iranian EFL textbooks and the primary focus of the present educational system is to make students pass tests and exams. According to Razmjoo \& Riazi (2006), Iranian schools lack the methodologies like Communicative Language Teaching, Community Language Learning, or Cooperative Learning. Since the sole focus of CLIL is not language and also a specific content, naturally a CLIL class involves high degrees of communication which in turn may help students develop oral communication skills.

\subsubsection{Deepen Awareness of Both Mother Tongue and Target Language}

On the contrary to the claim the above statement proposes, many parents might think that a CLIL class may hurt the children's first language. It seems that this is not the case. CLIL classes not only help students learn a second language -here English-but also help them gain a better understanding of their mother tongue as they naturally and subconsciously keep both languages alongside each other. Further, since CLIL is not supposed to be the main medium of teaching in schools and is seen as a complementary activity, students are given this opportunity to compare how content can be delivered in different languages thus they will find a better understanding of their own language.

\subsubsection{Develop Plurilingual Interests and Attitudes}

CLIL approach aims at developing students which can study and learn in at least on additional language- here English. Once such ability has been formed, it may encourage students to develop their abilities in other languages as well. Introducing different subjects through different languages naturally brings with itself some knowledge 
about the speakers of those languages. Since Iran is a relatively high mono-cultural environment, developing pluralingual attitudes may open new horizons to the Iranian students.

\subsubsection{Introduce a Target Language}

CLIL, in its simplest definition is teaching a specific content through an additional language. Therefore, it is quite obvious that in this case English -target language- will be widely introduced.

\subsection{The Content Dimension -CONTIX}

\subsubsection{Provide Opportunities to Study Content through Different Perspectives}

It has been mentioned earlier that learning a new language will provide learners with this opportunity to learn something from the speakers of that language and their culture. Mixing language with specific content not only enhances the cross-cultural understanding but also helps learners look at content through a different perspective as content-related materials in the target language may exhibit to some certain extents how teaching of that specific content is practiced where that specific language is used.

\subsubsection{Access Subject-specific Target Language Terminology}

Studying a specific content through another language - here English- will necessarily lead to learning the content-related terms in the target language. Therefore, students will be able to use extra-curricular materials which might be written and produced in the target language. This ability will have evident positive effects on the students' future academic success.

\subsubsection{Prepare for Future Studies and/or Working Life}

The number of Iranian students seeking higher education in English-speaking universities overseas is increasing. Introducing CLIL into Iranian educational system may serve as a preparation period for those who want to continue their education in other countries.

\subsection{The Learning Dimension - LEARNTIX}

\subsubsection{Complement Individual Learning Strategies}

Different learners have different learning styles which urge them to use different learning strategy. Since schools are required to follow a standardized program it is quite possible that this prescribed methodology does not suit a number of learners. Since in Iranian context school programs are dictated from a central authority and this prescribed system is not efficient at least in terms of English teaching, many students may not find the official school programs so effective. CLIL as a complementary program to the school standard program may provide students an opportunity to experience a new perspective toward learning different contents and subsequently foster more personalized learning procedures.

\subsubsection{Diversify Methods \& Forms of Classroom Practice}

CLIL adds a degree of variation to the conventional practice of teaching.

\subsubsection{Increase Learner Motivation}

Once the students have found that they can actually learn something through another language they may experience a higher level of motivation.

\section{CLIL Types, Focuses and Their Potential Applicability in Iranian Context}

As mentioned earlier in the previous section, the five dimensions of CLIL are actualized around some focal points. According to CLIL Compendium project, these focal points are as follow:

- $\quad$ Age-range of learners

- Socio-linguistic environment

- Degree of exposure to CLIL

Based on the factors of socio-linguistic and age-range of learners, CLIL Compendium proposes the following CLIL types:

- MONOLINGUAL 14-20 YEARS

- BILINGUAL 14-20 YEARS

- MULTILINGUAL 14-20 YEARS

- MONOLINGUAL 5-15 YeARS 
- $\quad$ BILINGUAL 5-15 YEARS

- MULTILINGUAL 5-15 YEARS

- MONOLINGUAL 3-6 YEARS

- $\quad$ BILINGUAL \& MULTILINGUAL 3-6 YEARS

Since the aim of this study is to propose an initial framework for incorporating CLIL approach into Iranian context and school system, the above list should be refined in order to reflect the Iranian context.

Iran is a monolingual country. By this we do not mean that there is no other existing language in this country. However, the principal mode of delivery in schools all over the country is Farsi which is the national language of Iran. In other words, it hardly ever happens that one can find classes within the country which consists of students with different languages. Even though in some regions and provinces across the country, for example Azerbaijan, Kurdistan, or southern provinces, people speak with some other languages alongside the national language, Farsi has such widespread acceptability that we can safely assume Iran as a monolingual context. Therefore, the abovementioned list might be initially refined to the following list:

- MONOLINGUAL 14-20 YEARS

- MONOLINGUAL 5-15 YEARS

- MONOLINGUAL 3-6 YEARS

Another possible refinement would be in case of age-range of learners. We propose that the age-range of learners is determined so that it could correspond to Iranian school system. In order to do so, we propose the second refinement would be done in the following way:

- MONOLOINGUAL 3-6 YEARS which corresponds to kindergarten and preschool ages

- MONOLINGUAL 7-11 YEARS which corresponds to elementary school ages

- MONOLINGUAL 12-14 YEARS which corresponds to secondary school ages

- MONOLINGUAL 15-18 YEARS which corresponds to high school ages

Considering a highly influential factor i.e. The Iranian National University Entrance contest which has a significant effect on the studying orientation in high schools years among Iranian students and even their parents which naturally results in establishing extra-curricular courses and classes which solely focus on improving testing skills, we believe that incorporating CLIL courses in this period might not work properly. Therefore, in order to achieve a workable framework, we propose the third level of refinement done in the following way:

- MONOLOINGUAL 3-6 YEARS

- MONOLINGUAL 7-11 YEARS

- MONOLINGUAL 12-14 YEARS

The other factor which has not been discussed here is degree of exposure to CLIL. According to CLIL Compendium Project, the degree of exposure to CLIL is defined as follows:

The proportion of CLIL teaching experienced by a learner in a school year

- $\quad$ Low - about $5-15 \%$ of teaching time

- Medium - about 15-50\% of teaching time

- High - over $50 \%$ of teaching time

Naturally as students progress to higher level of education, the number of subjects taught increases as well. The diversity of subjects potentially promises a richer context for employing CLIL approach. Therefore, we propose the following degrees of exposure to each CLIL type mentioned above:

- MONOLOINGUAL 3-6 YEARS, Low to Medium

- MONOLINGUAL 7-11 YEARS, Medium

- MONOLINGUAL 12-14 YEARS, Medium to High

The abovementioned list means that the incorporation of CLIL may start from a set of limited hours in kindergarten and preschools and gradually increases to higher amounts of exposure in elementary and secondary school. 


\section{Conclusion}

CLIL is of evident potentiality for Iranian context. Since internationalization is becoming an emerging trend all over the globe, each nation should be equipped with the right tools to be prepared to deal with this global orientation.

CLIL has experienced most of the research in European context mostly through EU and European Commission funded projects. Though these projects mostly manifest and reflect cultural and socio-linguistic characteristics of European countries, we believe that they may provide us with invaluable insights about incorporating such an approach into different local contexts especially those of developing countries which seek to find their position in the international community.

This study is an initial attempt to localize European-led projects in the field of CLIL in the Iranian context.

It is strongly suggested that scholars and researchers in the field of TEFL in Iran pay much more attention to the potentialities of CLIL. As mentioned earlier, any attempt to establish such a trend is a strategic investment.

\section{References}

Ghorbani, M. R. (2009). ELT in Iranian high schools in Iran, Malaysia and Japan: Reflections on how tests influence use of prescribed textbooks. Reflections on English Language Teaching, 8(2), 131-139.

Jahangard, A. (2007). Evaluation of EFL materials taught at Iranian public high schools. The Asian EFL Journal, 9(2), 130-150.

Lang, J. (2002). Foreword by Minister of Education, France in TIE_CLIL Professional Development Course. TIE-CLIL: Milan.

Marsh, D. (1994). Bilingual Education \& Content and Language Integrated Learning. International Association for Cross-cultural Communication, Language Teaching in the Member States of the European Union (Lingua) University of Sorbonne. Paris.

Marsh, D. (2002). CLIL/EMILE - The European Dimension: Actions, Trends and Foresight Potential. DG Education \& Culture, European Commission.

Marsh, D. et al. (2001). Integrating Competencies for Working Life. UNICOM: Jyväskylä.

Marsh, D., \& Langé, G. (2000). Using Languages to Learn and Learning to Use Languages. TIE-CLIL: Jyväskylä \& Milan.

Ostovar Namaghi, S. A. (2006). Forces Streering Iranian Language Teachers' Work: A Ground Theory. The Reding Matrix, 6(2), September.

\section{Websites}

http://www.clilcompendium.com

http://archive.ecml.at

http://www.cilt.org.uk/secondary/14-19/intensive_and_immersion/clil.aspx 